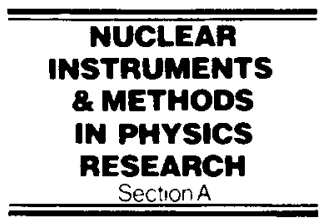

\title{
A constrained dual-energy reconstruction method for material-selective transmission tomography is
}

\author{
Neal H. Clinthorne * \\ Diviston of Nuclear Medicine, 3480 Kresge III, Box 0552, The University of Michigan, Ann Arbor, Michtgan 48109-0552, USA
}

\begin{abstract}
A new reconstruction method has been developed for material-selective tomography that allows non-negativity and maximum density constraints to be enforced on each pixel. In addition, uncertainty in the projection data is appropriately accounted for. Results of applying the constrained reconstruction to simulated data demonstrate significant improvements over unconstrained reconstructions.
\end{abstract}

Dual energy X-ray transmission tomography can be useful for estimating attenuation coefficients for PET and SPECT imaging [1]. In an effort to minimize the dose to the patient in the transmission scan, we are investigating new reconstruction approaches that allow reduced X-ray exposure for a given error in attenuation estimates.

As in Ref. [2], we consider the object to be composed to two basis materials. However, instead of allowing all

\footnotetext{
${ }^{4}$ Full paper submitted to Nucl. Instr. and Meth. A. Research supported under NIH grants RO1 CA54362 and RO1 CA32846.

* Tel. +1 313763 9244, fax +1 313764 0288, e-mail clinthorne@petclu.petnet.med.umich.edu.
}

linear combinations of the basis vectors, we constrain the feasible attenuation values to only convex combinations, which corresponds to non-negative densities of each material. Further performance enhancements are obtained by restricting the maximum allowable density of each material. Imposing these physically meaningful constraints stabilizes the reconstruction error as the X-ray exposure (and hence dose) is reduced. Conventional reconstruction techniques neither impose these constraints nor correctly weight uncertainty in the projection data.

We solve the reconstruction problem by minimizing an uncertainty-weighted, penalized least-squares objective function parameterized by the unknown material densities. A grouped coordinate descent modification to the sequentially updating Gauss-Seidel algorithm used by Sauer and

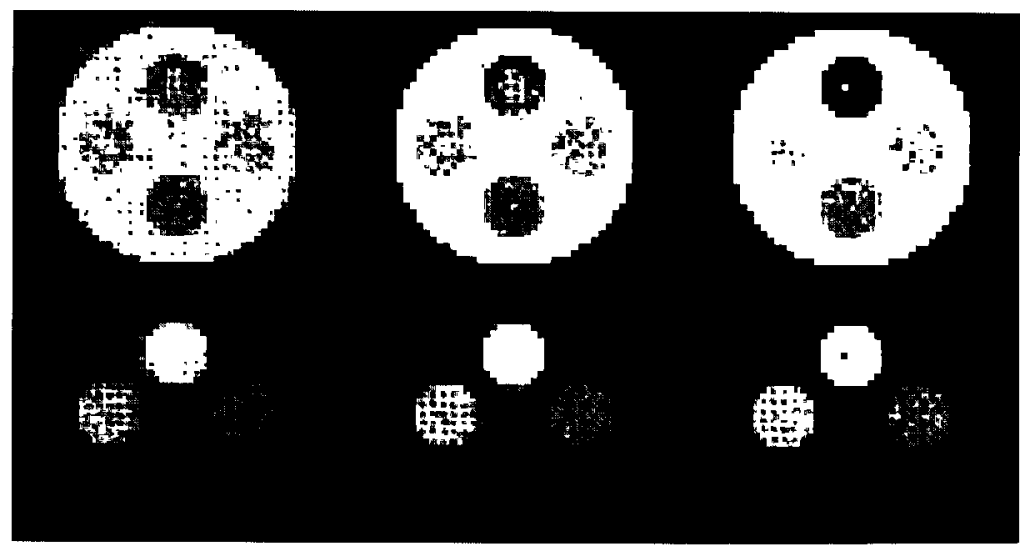

Fig. 1. Reconstructions of soft-tıssue (top row) and bone (bottom row) densities for simulated tomographic data. Left: unconstrained reconstruction. Middle: non-negativity constraints only Right: non-negativity and maximum density constraints. True soft-tissue densities: background, $1.0 \mathrm{~g} / \mathrm{cm}^{3}$. Regions clockwise starting from 12 o'clock, $0 \mathrm{~g} / \mathrm{cm}^{3}, 05 \mathrm{~g} / \mathrm{cm}^{3}, 0.2 \mathrm{~g} / \mathrm{cm}^{3}, 0.5 \mathrm{~g} / \mathrm{cm}^{3}$. True bone densities: background, $0 \mathrm{~g} / \mathrm{cm}^{3}$. Regions in same order as previous, $2.0 \mathrm{~g} / \mathrm{cm}^{3}, 0.5 \mathrm{~g} / \mathrm{cm}^{3}, 0 \mathrm{~g} / \mathrm{cm}^{3} 1.0 \mathrm{~g} / \mathrm{cm}^{3}$. 
Bouman [3] is employed for the iterative solution. Each pair of pixels, corresponding to a single spatial location, is updated by choosing new values from the constraint set to minimize the objective. A single iteration is complete when all pixels have been updated in this manner.

The method has been tested using simulated dual-energy tomographic data for soft-tissue and bone at 40 and $80 \mathrm{keV}$. Results are shown in Fig. 1 where unconstrained reconstructions, those employing only non-negativity constraints, and the reconstructions using both non-negativity and maximum density constraints are shown left-to-right respectively. The advantage of the constraints is clearly seen when the densities lie on the boundary of the constraint set (all regions except the one at 3 o'clock). If the density is in the interior of the constraint set, the improvement is not as dramatic. Nevertheless, with the exception of lung tissue, most body tissues-being either soft-tissue or soft-tissue/bone mixtures-will be at or near the maximum feasible density, and it is precisely this situation that favors the fully constrained reconstruction over alternatives.

\section{References}

[1] B.H. Hasegawa, T F. Lang, J.K. Brown, E.L. Gingold and S.C. Blankespoor, Conf. Record of the 1992 IEEE Nuclear Science Symp. and Medical Imaging Conf., 1993, p. 1059.

[2] R.E. Alvarez and A. Macovski. Phys. Med. Biol 21 (1976) 733.

[3] K. Sauer and C. Bouman, IEEE Trans. Sig Proc. 41(2) (1993) 534. 